\title{
Eficiência fotoquímica de gramíneas forrageiras tropicais submetidas à deficiência hídrica ${ }^{1}$
}

\author{
Manoel Messias Pereira da Silva ${ }^{2}$, Hernán Maldonado Vasquez ${ }^{3}$, Ricardo Bressan-Smith ${ }^{4}$, José \\ Fernando Coelho da Silva ${ }^{3}$, Eleonora D’Avila Erbesdobler ${ }^{5}$, Paulo Sérgio Cruz de Andrade Junior ${ }^{6}$ \\ ${ }^{1}$ Parte do trabalho de tese de Doutorado do primeiro autor, financiado com recursos da Fundação de Amparo à Pesquisa do Estado do Rio \\ de Janeiro (FAPERJ). \\ 2 UPIS - Brasilia, DF. \\ ${ }^{3}$ LZNA/CCTA/UENF. \\ 4 Setor de Fisiologia Vegetal, LMGV/CCTA/UENF. \\ ${ }^{5}$ Laboratório de Ciências Ambientais (LCA), Centro de Biociências e Biotecnologias (CBB), UENF. \\ ${ }^{6}$ Médico Veterinário.
}

RESUMO - Neste estudo, as espécies capim-setária (Setaria anceps Stapf.), capim-hemárthria (Hemarthria altissima [Poir] Stapf. \& Hubbard), capim-do-nilo (Acroceras macrum Stapf.) e capim-angola (Brachiaria purpurascens [Raddi] Henr.) foram submetidos à deficiência hídrica moderada a seca com o objetivo de avaliar o efeito do déficit hídrico sobre as características de fluorescência da clorofila. O experimento foi realizado em casa de vegetação, em blocos ao acaso, constituídos pelas quatro espécies cultivadas sob dois regimes hídricos (irrigado e não-irrigado), em três repetições. Durante a indução da deficiência hídrica, foram monitoradas as seguintes variáveis de fluorescência da clorofila $a$ : fluorescência inicial ( $\left.\mathrm{F}_{0}\right)$, fluorescência máxima $\left(\mathrm{F}_{\mathrm{m}}\right)$, rendimento quântico máximo do PSII $\left(\mathrm{F}_{\mathrm{v}} / \mathrm{F}_{\mathrm{m}}\right)$, taxa relativa de transporte de elétrons (ETR), quenching fotoquímico (qP) e quenching não fotoquímico (qN). Após dez dias de submissão ao estresse hídrico, os valores de $F_{o}$ e $F_{m}$ decresceram em todas as espécies. Os menores valores de Fv/Fm foram encontrados no capim-do-nilo e no capimsetária, o que configura baixo rendimento fotoquímico nessas espécies sob déficit hídrico, ao contrário do capim-hemárthria, que apresentou valores similares em ambos os tratamentos. Os valores mais altos de qN foram registrados no capim-hemárthria e no capim-angola, demonstrando boa capacidade dessas espécies em dissipar a energia luminosa absorvida em excesso para manter baixo estado de redução nos aceptores primários de elétrons do PSII. Em geral, as curvas de ETR em resposta ao fluxo de fótons fotossintéticos mostraram-se distintas entre os tratamentos, sendo mais pronunciadas no capim-do-nilo. Nessa espécie, a eficiência dos transportadores de elétrons do PSII foi seriamente comprometida pelo déficit hídrico.

Palavras-chave: fluorescência da clorofila $a$, fotossíntese, fotossistema II, taxa relativa de transporte de elétrons

\section{Photochemical efficiency of tropical forage grasses submitted to water deficit}

\begin{abstract}
In this study, individuals of setariagrass (Setaria anceps Stapf.), limpograss (Hemarthria altissima [Poir] Stapf. \& Hubbard), nilograss (Acroceras macrum Stapf.) and angola grass (Brachiaria purpurascens [Raddi] Henr.) were submitted to moderate drought. The characteristics of chlorophyll $a$ fluorescence (inicial fluorescence, Fo; maximum fluorescence, Fm; photochemistry efficiency, Fv/F-m; photochemical quenching, qP; nonphotochemical quenching, $\mathrm{qN}$ and relative electron transport rate, ETR) were investigated in an experiment carried out in greenhouse, using plastic pots. The experimental treatments were allocated in a randomized complete blocks design, with three replications. The values of Fo and Fm decreased in all species after 10 days of drought, this effect being more evident in nilograss and setariagrass. The Fv/Fm values for nilograss and setariagrass decreased dramatically while that one for hemarthriagrass did not differ from the control. This results suggest a lower photochemical efficiency of photosynthesis in nilograss and setariagrass under water stress compared to hemarthriagrass and to the hea,lthy plants. The highest values of qN were observed for hemarthriagraas and angolagrass. This showed that the increased capacity to dissipate the excessive energy to drive photosynthesis was satisfactory to maintain a low reduction state of the primary electron acceptor of the photosystem II (measured as qP). In general, ETR curves in response to increasing photosynthetic photon flux differed from control to stressed plants, especially for nilograss. In this species, a serious damage caused by water stress provoked a significant reduction to the efficiency of the electron transporters of PSII.
\end{abstract}

Key Words: chlorophyll a fluorescence, electron transport relative rate, photosynthesis, photosystem II 


\section{Introdução}

O elevado potencial de produção primária das espécies de gramíneas perenes de via fotossintética $\mathrm{C} 4$, em comparação às $\mathrm{C} 3$, tem sido atribuído às condições de regiões tropicais, em baixas latitudes, de maior insolação e mais alta temperatura, e à melhor eficiência no uso da água (Rodrigues \& Rodrigues, 1994). Entretanto, gramíneas tropicais podem sofrer limitações ambientais, de modo que a presença da água em condições ideais é determinante para o crescimento e desenvolvimento vegetal. A deficiência hídrica provocada pela seca estacional influencia a relação entre a água e as trocas gasosas nas gramíneas forrageiras tropicais. Segundo Kaiser (1987), o estresse hídrico pode causar severa inibição da fotossíntese, mesmo em plantas $\mathrm{C} 4$, sobretudo em razão da maior resistência difusiva à entrada do $\mathrm{CO}_{2}$. Esses eventos são oriundos da redução da turgescência das células-guarda do estômato, seguida pelo fechamento do poro estomático (Baruch, 1994; Silva et al., 2001).

Embora as medidas de trocas gasosas $\left(\mathrm{CO}_{2} \mathrm{e} \mathrm{O}_{2}\right)$ sejam importantes para determinar as taxas fotossintéticas, podem não ser eficientes para avaliar os efeitos deletérios nos cloroplastos, ocasionados pelas diversas formas de estresse abiótico (Durães, 2003). Uma alternativa às medidas de trocas gasosas é a avaliação da eficiência fotoquímica da fotossíntese, obtida por meio das diversas variáveis da fluorescência da clorofila $a$. O rendimento da fluorescência da clorofila revela o nível de excitação da energia no sistema de pigmentos que dirige a fotossíntese e fornece subsídios para estimar a inibição ou o dano no processo de transferência de elétrons do fotossistema II (PSII) (BolhàrNordenkampf et al., 1989). Além disso, é uma técnica rápida, não-destrutiva e sensível, constituindo importante avanço em estudos fisiológicos e em ecologia de plantas (Krause \& Weiss, 1991).

Condições extremas de estresse ambiental podem afetar os eventos no tilacóide, interferindo na eficiência da fotossíntese e inativando o fotossistema II (P680) e a cadeia de transporte de elétrons que daria origem ao ATP e NADPH (Krause \& Weiss, 1991; Costa et al., 2003).

As principais variáveis observadas nas medições da fluorescência da clorofila $a$ são: fluorescência inicial $\left(\mathrm{F}_{0}\right)$, fluorescência máxima $\left(\mathrm{F}_{\mathrm{m}}\right)$, fluorescência variável $\left(\mathrm{F}_{\mathrm{v}}\right)$, rendimento quântico máximo do PSII $\left(\mathrm{F}_{\mathrm{v}} / \mathrm{F}_{\mathrm{m}}\right)$, taxa relativa de transporte de elétrons (ETR), quenching fotoquímico (qP) e quenching não-fotoquímico $(\mathrm{qN}) . \mathrm{O}_{0}$ representa a fluorescência com todos os centros de reação "abertos" e refere-se à emissão de fluorescência pelas moléculas de clorofila $a$ do complexo coletor de luz do PSII (Krause \&
Weiss, 1991). O $\mathrm{F}_{\mathrm{m}}$ indica a completa redução da quinona $\mathrm{A}\left(\mathrm{Q}_{\mathrm{A}}\right)$ a partir da incidência de um pulso de luz no centro de reação $Q_{A}$, gerando fluorescência máxima. A diferença entre $F_{m}$ e $F_{0}$ resulta na fluorescência variável $\left(F_{v}\right)$. A $F_{v}$ representa o fluxo de elétrons do centro de reação do PSII (P680) até a plastoquinona $\left(\mathrm{PQH}_{2}\right)$. O rendimento quântico máximo é calculado como: $\mathrm{F}_{\mathrm{v}} / \mathrm{F}_{\mathrm{m}}=\left(\mathrm{F}_{\mathrm{m}}-\mathrm{F}_{0}\right) / \mathrm{F}_{\mathrm{m}}$. Quando uma planta está com seu aparelho fotossintético intacto, a razão $\mathrm{F}_{\mathrm{v}} / \mathrm{F}_{\mathrm{m}}$ deve variar entre 0,75 e 0,85 (BolhàrNordenkampf et al., 1989), enquanto queda nesta razão reflete a presença de dano fotoinibitório nos centros de reação do PSII (Björkman \& Demming, 1987). O quenching fotoquímico (qP) indica a proporção dos centros de reação do PSII que estão abertos, enquanto o quenching não-fotoquímico (qN) refere-se ao nível de fluorescência máxima $(\mathrm{Fm})$ no qual a eficiência fotoquímica está no máximo e a dissipação térmica está no mínimo (Maxwell \& Johnson, 2000).

Nesse estudo, todas as gramíneas forrageiras avaliadas são consideradas tolerantes a ambientes sujeitos a inundação periódica, provavelmente em razão do eficiente sistema de difusão de oxigênio na raiz e no caule. Embora a maioria dos estudos seja direcionada à manutenção da produtividade em ambientes alagados, muitas dessas áreas podem estar sujeitas a secas estacionais, comprometendo significativamente o crescimento e, em alguns casos, causando a morte dessas gramíneas (Armstrong et al., 1991)

O monitoramento da fluorescência da clorofila $a$ nas gramíneas forrageiras pode ser útil na identificação da capacidade de tolerância dessas espécies à deficiência hídrica. Desse modo, objetivou-se verificar os danos provocados pelo déficit hídrico do solo sobre o fotossistema II, por meio da observação das características de fluorescência da clorofila $a$, em quatro gramíneas forrageiras tropicais: capim-setária (Setaria anceps Stapf.), capimhemárthria (Hemarthria altissima [Poir] Stapf. \& Hubbard), capim-do-nilo (Acroceras macrum Stapf.) e capim-angola (Brachiaria purpurascens [Raddi] Henr.).

\section{Material e Métodos}

Plantas das espécies capim-setária (Setaria anceps Stapf.), capim-angola (Brachiaria purpurascens [Raddi] Henr.), capim-hemárthria (Hemarthria altissima [Poir.] Stapf \& Hubbard) e capim-do-nilo (Acroceras macrum Stapf.) foram submetidas a diferentes condições hídricas para avaliação de fatores fotoquímicos da fotossíntese.

O ensaio foi conduzido em casa de vegetação com cobertura de lona plástica transparente, na Unidade de 
Apoio à Pesquisa (UAP) do Centro de Ciências e Tecnologias Agropecuárias (CCTA) da Universidade Estadual do Norte Fluminense (UENF), em Campos dos Goytacazes, RJ.

Em julho de 2002, mudas enraizadas das quatro espécies, retiradas de plantas existentes no Setor de Forragicultura do LZNA, nas dependências do Colégio Agrícola Estadual “Antônio Sarlo", em Campos dos Goytacazes, RJ, foram plantadas em vasos plásticos, com furos, com capacidade para $6 \mathrm{dm}^{3}$ de solo, apoiados em pratos plásticos. O solo para enchimento dos vasos, classificado como Latossolo Amarelo Distrófico e procedente do Setor de Forragicultura do LZNA, foi passado em peneiras com malhas de $4 \mathrm{~mm}$, retirando-se amostras para análises no Laboratório de Análises Químicas de Solo da Universidade Federal Rural do Rio de Janeiro, Campus Dr. Leonel Miranda, em Campos dos Goytacazes, RJ. O resultado da análise do solo (Tabela 1) indicou a necessidade de correção da acidez por meio da elevação da saturação de bases em 50\% (Werner et al., 1986).

Após o preparo do solo, encheram-se os vasos e plantaram-se as mudas das gramíneas, realizando-se a pesagem individual das mudas e dos respectivos vasos preenchidos com solo, com o objetivo de controlar o peso de cada vaso para posterior aplicação da irrigação. A adubação básica do solo para cada vaso foi feita com $110 \mathrm{mg} / \mathrm{dm}^{3}$ de N (sulfato de amônio), $150 \mathrm{mg} / \mathrm{dm}^{3}$ de P (superfosfato simples) e $50 \mathrm{mg} / \mathrm{dm}^{3}$ de K (cloreto de potássio). Após o plantio e durante o estabelecimento das mudas nos vasos, foram realizadas irrigações com água de torneira, controlando-se a quantidade de água aplicada em cada vaso por meio da pesagem individual, de modo a manter a umidade do solo em $80 \%$ de saturação relativa à porosidade total (Kiehl, 1979).

Em agosto de 2002, após o estabelecimento das plantas, efetuou-se o corte de uniformização das quatro gramíneas, a $5 \mathrm{~cm}$ de altura do solo, utilizando-se uma "tesoura-podão", para obtenção de novos perfilhos a partir das gemas basais. Em torno de uma semana após o surgimento dos perfilhos basais, foram aleatoriamente identificados três perfilhos em cada vaso (com o uso de anéis feitos de fios de náilon coloridos), destinados à avaliação das gramíneas.
O delineamento experimental utilizado foi o de blocos ao acaso, constituído de dois regimes hídricos (irrigado e não-irrigado) e quatro espécies de gramíneas, com três repetições, totalizando 24 vasos (12 irrigados +12 nãoirrigados). Cada bloco apresentava plantas com tamanho uniforme (para cada espécie) e cada vaso ou unidade experimental possuía um perfilho marcado. No tratamento irrigado (controle), os vasos foram mantidos com rega diária, a 100\% da saturação relativa da porosidade total do solo, enquanto, no tratamento não-irrigado, o experimento se iniciou com a suspensão da rega e se encerrou dez dias depois, quando a maioria das plantas apresentava sintomas de murcha e enrolamentos acentuados. A quantidade de água $(\mathrm{Qw})$ adicionada por tratamento foi determinada pela fórmula $\mathrm{Qw}$ $=$ volume do solo $\mathrm{x}$ porosidade total $\left(\mathrm{P}_{\mathrm{T}}\right)$. A porosidade total $\left(\mathrm{P}_{\mathrm{T}}\right)$ do solo foi calculada considerando-se a densidade do solo (ds) e a densidade de partícula (dp), que envolvem a massa e o volume de solo, de acordo com a seguinte fórmula: $\mathrm{P}_{\mathrm{T}}=1$ - (ds/dp), conforme a metodologia descrita por Kiehl (1979). Durante o período experimental, a água perdida por evapotranspiração era reposta diariamente utilizando-se o método gravimétrico, com o uso de balança, a partir do conhecimento prévio dos pesos de cada vaso na implantação dos tratamentos.

Em setembro de 2002, após 45 dias da rebrota, um perfilho basal (abaixo da altura de corte) e maduro, previamente marcado em cada vaso, foi utilizado para as avaliações da fluorescência da clorofila $a$. Em cada perfilho marcado, a primeira folha totalmente expandida a partir do ápice (presença de lígula completamente exposta) foi devidamente identificada com fios de lã vermelha, para as devidas avaliações. As variáveis da fluorescência da clorofila $a$ foram determinadas com o fluorímetro portátil de luz modulada MINI-PAM (Walz, Germany), a partir do qual foram obtidas a fluorescência inicial $\left(\mathrm{F}_{0}\right)$, a fluorescência máxima $\left(\mathrm{F}_{\mathrm{m}}\right)$, o rendimento quântico máximo do PSII $\left(\mathrm{F}_{\mathrm{v}} / \mathrm{F}_{\mathrm{m}}\right)$, a taxa relativa de transporte de elétrons (ETR), $o$ quenching fotoquímico (qP) e o quenching não-fotoquímico (qN). As mensurações foram feitas com o auxílio de pinças de metal (DLC-8) acopladas ao sensor MINI-PAM, colocadas

Tabela 1 - Resultados da análise química do solo

Table 1 - Results of soil chemical analysis

\begin{tabular}{|c|c|c|c|c|c|c|c|c|c|c|c|c|c|}
\hline $\mathrm{pH}$ & $\mathrm{P}$ & $\mathrm{mg} / \mathrm{dm}^{3} \quad \mathrm{~K}$ & & $\mathrm{Ca}$ & & $\mathrm{Mg}$ & \multirow{2}{*}{\multicolumn{2}{|c|}{$\mathrm{cmol}_{\substack{\mathrm{c} / \mathrm{dm}^{3 *} \\
0,7}}^{\mathrm{Al}}$}} & $\mathrm{H}+\mathrm{Al}$ & $\mathrm{Na}$ & & $\begin{array}{r}\mathrm{C} \\
\%\end{array}$ & $\begin{array}{c}\mathrm{MO} \\
\mathrm{g} / \mathrm{dm}^{3}\end{array}$ \\
\hline 4,6 & 1 & 10 & & 0,5 & & 0,6 & & & 3,0 & \multirow{3}{*}{${ }_{1,0} \begin{array}{l}0,09 \\
\mathrm{mg} / \mathrm{dm}^{3}\end{array}$} & & 0,35 & 6,0 \\
\hline S.B. & $\mathrm{T}$ & \multirow{2}{*}{$\mathrm{cmol}_{1,9}^{\mathrm{t}} \mathrm{c}^{/ \mathrm{dm}^{3}}$} & $\mathrm{~m}$ & & $\mathrm{~V}$ & & $\mathrm{Fe}$ & & & & $\mathrm{Mn}$ & S & B \\
\hline 1,2 & 4,2 & & 37 & & 29 & & 5,0 & & & & 1,5 & 83,1 & 0,32 \\
\hline
\end{tabular}

Centimo carga/dm ${ }^{3}$ de solo (Centimol charge $/ \mathrm{dm}^{3}$ of soil). 
na região mediana das folhas, em um dos lados do limbo foliar, evitando-se a nervura central.

Antes de cada determinação individual, uma porção da folha foi mantida no escuro, ou seja, permaneceu com a pinça fechada por pelo menos 30 minutos para que todos os centros de reação naquela região da folha adquirissem a condição de "abertos" (extintor fotoquímico não-reduzido). As leituras para obtenção das variáveis $\mathrm{F}_{0}, \mathrm{~F}_{\mathrm{m}}$ e $\mathrm{F}_{\mathrm{v}} / \mathrm{F}_{\mathrm{m}}$ foram realizadas entre o $1^{\mathrm{O}}$ e $10^{\mathrm{O}}$ dias sem irrigação (total de cinco determinações), a cada dois dias, com pulsos de luz saturante de $0,3 \mathrm{~s}$, para promover o fechamento dos centros de reação do PSII (extintor fotoquímico reduzido), enquanto as variáveis ETR, $\mathrm{qP}$ e qN foram determinadas em uma curva de resposta à luz, com fluxo de fótons fotossintéticos (FFF) de 0 a $2291 \mathrm{mmol} \mathrm{m}^{-2} \mathrm{~s}^{-1}$, por um período inferior a dois minutos. Todas as leituras foram realizadas entre as $15 \mathrm{e} 17 \mathrm{~h}$ e os dados de fluorescência obtidos foram automaticamente armazenados no MINI-PAM e, posteriormente, transferidos para um computador utilizando-se o programa Wincontrol.

As análises de variância e a comparação de médias pelo teste Tukey a $5 \%$ foram realizadas utilizando-se o programa SAEG (Sistemas para Análises Estatísticas e Genéticas), desenvolvido pela Universidade Federal de Viçosa (Euclydes, 1983).

\section{Resultados e Discussão}

O acompanhamento das variáveis da fluorescência da clorofila $a$ conforme a diminuição da umidade do solo, em geral, mostrou diferenças entre as quatro gramíneas avaliadas neste estudo. À medida que o déficit hídrico se acentuava, com o decorrer dos dias, foi evidente a redução nos valores de $F_{0}, F_{m}$ e $F_{v} / F_{m}$, nas quatro espécies (Figura 1).

Os valores de $\mathrm{F}_{0}$ apresentaram os maiores percentuais de redução no capim-do-nilo, com reduções de 33,7 a 69,2\% na primeira e última avaliações em relação à condição inicial, respectivamente. Os capins hemárthria, angola e setária apresentaram comportamento semelhante durante os 10 dias de suspensão da irrigação, com redução do $F_{0}$ para essas gramíneas entre 36,3 e 39,1\% ao final das avaliações (décimo dia), que pode ser indicativo da destruição do centro de reação do PSII (Bolhàr-Nordenkampf et al., 1989) e que a queda mais acentuada desta variável no capim-donilo poderia indicar menor possibilidade de adaptação às condições fotoinibitórias provenientes do déficit hídrico.

Os percentuais de redução de $\mathrm{F}_{\mathrm{m}}$ nos capins do-nilo e setária foram relativamente superiores aos encontrados nas demais gramíneas (Figura 1), registrando-se acentuada queda com o avanço dos dias sem irrigação e ao décimo dia

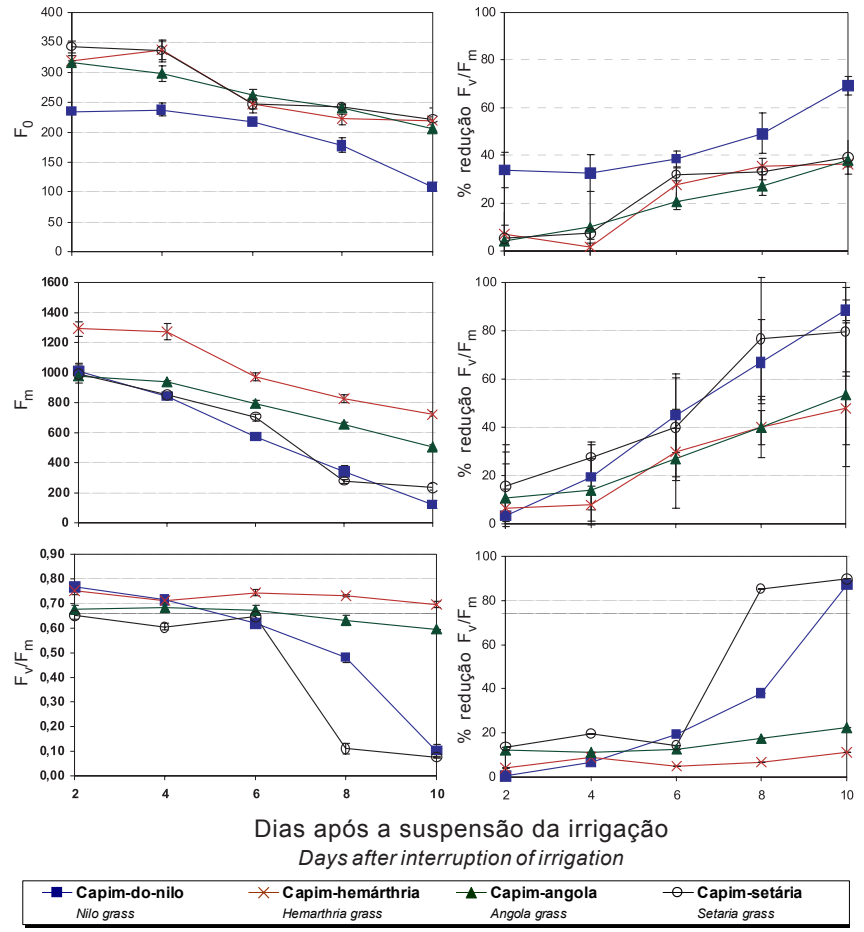

Figura 1 - Curvas das variáveis de fluorescência da clorofila a: fluorescência inicial $\left(F_{0}\right)$, fluorescência máxima $\left(F_{m}\right)$ e rendimento quântico máximo $\left(F_{v} / F_{m}\right)$ e suas respectivas variações percentuais sobre as condições iniciais, determinadas em quatro espécies de gramíneas forrageiras tropicais sob déficit hídrico de até dez dias. As barras representam o erro-padrão da média de três repetições.

Figura 1 - Curves of chlorophyll a fluorescence parameters: initia fluorescence $\left(F_{0}\right)$, maximum fluorescence $\left(F_{m}\right)$ and ratio $F_{v} / F_{m}$, and respective percentage variations, determinated in four tropical forage grass, during ten days without irrigation. The bars representing the standard-error of mean of three replicates.

de avaliação (de 79,6 e 88,4\%, para o capim-setária e o capim-do-nilo, respectivamente). Esse comportamento indica uma resposta negativa ao constante decréscimo da água disponível no solo, culminando com o dano irreversível aos tecidos foliares (senescência). Por outro lado, nos capins hemárthria e angola, a redução do valor de $\mathrm{F}_{\mathrm{m}}$ com o avanço do déficit hídrico foi, aproximadamente, a metade da observada no capim-do-nilo, de 47,6 e $53,5 \%$, respectivamente.

Ressalta-se que à medida que a disponibilidade de água para a planta diminui, a transpiração é reduzida drasticamente, potencializando outros fatores ambientais de estresse, como temperatura, tornando-se mais difícil separar os efeitos de alta temperatura daqueles de déficit hídrico (Gates, 1968). Admite-se, portanto, que a intensificação dos efeitos degenerativos decorrentes da deficiência hídrica sobre os tecidos vegetais pode ocasionar danos permanentes na planta, culminando com sua morte (Larcher, 1995). Esse 
efeito foi observado a partir do quarto dia após a suspensão da irrigação dos vasos. Verificou-se ainda que, a partir deste período, todas as gramíneas já apresentavam enrolamento foliar com o amarelecimento dos ápices das lâminas foliares em alguns casos. Silva et al. (2001) observaram enrolamento foliar e murchamento severo em capim-canarana (Echinochloa pyramidalis) e capim-setária (Setaria anceps) cultivados em vasos e submetidos a déficit hídrico por 12 dias. O enrolamento foliar pode ser uma estratégia para redução da transpiração, com o objetivo de manter os estômatos em condições de elevada umidade. Nesse estudo, o enrolamento foliar foi inicialmente observado nas plantas de capim-do-nilo.

A diminuição dos valores absolutos de $\mathrm{F}_{\mathrm{m}}$ caracteriza deficiências de fotorredução da quinona $\mathrm{A}\left(\mathrm{Q}_{\mathrm{A}}\right)$, que podem estar associadas à inativação do PSII nas membranas tilacoidais, afetando diretamente o fluxo de elétrons entre os fotossistemas. Os capins hemárthria e angola apresentaram os menores percentuais de redução de $\mathrm{F}_{\mathrm{m}}$ ao longo do período após a suspensão da irrigação, o que sugere menor susceptibilidade dessas espécies à maior intensidade e duração do déficit hídrico aplicado a todas as plantas do estudo. Por outro lado, a redução da $\mathrm{F}_{\mathrm{m}}$ foi maior nos capins do nilo e setária, indicando maior susceptibilidade à intensificação do estresse hídrico. As diferenças de valores de $\mathrm{F}_{\mathrm{m}}$ entre as espécies podem demonstrar variações nas propriedades dos aceptores de elétrons do PSII, causadas por mudanças conformacionais induzidas pelo estresse no principal constituinte do complexo protéico que forma o PSII, a proteína D1 (Bulkhov at al., 1999). Assim, a perda de atividade do PSII nas espécies susceptíveis está provavelmente associada à diminuição da capacidade de reoxidação das quinonas, resultando em menor eficiência no transporte de elétrons para o PSI (De Las Rivas \& Barber, 1997).

O rendimento quântico máximo do PSII, estimado pela razão $\mathrm{F}_{\mathrm{v}} / \mathrm{F}_{\mathrm{m}}$, indica a dissipação fotoquímica de energia e expressa a eficiência de captura desta energia de excitação pelos centros de reação abertos do PSII (Baker, 1991; Krause \& Weis, 1991). Segundo Bolhàr-Nordenkampf et al. (1989), quando uma planta não está submetida ao estresse, a razão $F_{v} / F_{m}$ deve ser de 0,75 a 0,85 . Os valores referentes à razão $\mathrm{F}_{\mathrm{v}} / \mathrm{F}_{\mathrm{m}}$ durante os dez dias sem irrigação, para as quatro espécies estudadas, são apresentados na Tabela 2. Os maiores valores $(\mathrm{P}<0,05)$ foram observados nos capins hemárthria e angola, com destaque para o hermárthria, que manteve a razão $\mathrm{F}_{\mathrm{v}} / \mathrm{F}_{\mathrm{m}}$ próxima ao limite inferior de $0,75 \mathrm{e}$, em relação ao tratamento não-irrigado, apresentou redução da razão $F_{v} / F_{m}$ de apenas $11,0 \%$ no décimo dia sem irrigação, aproximadamente metade da observada no capimangola $(22,5 \%)$ no mesmo período. Esse comportamento poderia indicar dano menos severo ao PSII para o capimhemárthria sob déficit hídrico, enquanto o capim-do-nilo e o capim-setária tiveram queda mais acentuada na razão $\mathrm{F}_{\mathrm{v}} / \mathrm{F}_{\mathrm{m}}$ a partir do sexto dia de suspensão da irrigação, com reduções de 87 e 90\%, respectivamente, ao final da avaliação, indicando efeito mais severo do estresse e a maior susceptibilidade dessas duas espécies (Figura 1). O declínio da razão $\mathrm{F}_{\mathrm{v}} / \mathrm{F}_{\mathrm{m}}$ em plantas submetidas a estresse ambiental, hídrico ou térmico, indica a ocorrência de dano fotoinibitório nos centros de reação do PSII (Björkman \& Demming, 1987) que, no caso do capim-do-nilo, foi maior $(\mathrm{P}<0,05)$ que nos capins hemárthria e angola, mas não diferiu $(\mathrm{P}>0,05)$ do capim-setária no décimo dia sem irrigação (Tabela 2).

Os resultados observados para as variáveis de fluorescência nas quatro gramíneas submetidas ao déficit hídrico podem indicar que o capim-hemárthria apresenta aparelho fotossintético mais eficiente que os demais quanto à tolerância às condições fotoinibitórias decorrentes da baixa disponibilidade de água no solo. Entretanto, a manutenção dos valores $\mathrm{F}_{\mathrm{v}} / \mathrm{F}_{\mathrm{m}}$ observados em capim-hemárthria ao longo do período de deficiência hídrica também poderia estar associada à sua morfologia, cujas principais características são sistema radicular extenso, hastes decumbentes, e folhas longas e finas (Alcântara \& Bufarah, 1992). Estas características podem contribuir para que o capim-hemárthria apresente menores perdas hídricas, diferentemente das gramíneas de porte ereto, como o capim-do-nilo e o capimsetária, que apresentam características de área e arquitetura foliar diferenciadas das gramíneas decumbentes (Drew et al., 1979; Rhind \& Goodenough, 1979).

Ao $10^{0}$ dia após a suspensão da irrigação, houve redução do ponto de saturação dos carreadores de elétrons no capim-angola e capim-do-nilo, especialmente no capimdo-nilo (Figura 2). Apesar disso, a razão $\mathrm{F}_{\mathrm{v}} / \mathrm{F}_{\mathrm{m}}$ do capimangola $(0,595)$ não foi tão reduzida como a do capim-do-nilo $(0,100)$ (Tabela 2$)$, indicando que o capim-angola pode apresentar eficiente sistema de dissipação energética do tipo não-fotoquímica, provavelmente influenciada pela presença de zeaxantina, cuja ação fotoprotetora ocorre nos complexos coletores de luz do PSII (Horton et al., 1996).

Segundo Mohammed et al. (1995), o ETR aumenta conforme a intensidade de luz, até que ocorra a saturação dos carreadores de elétrons. Em alguns casos, a curva de ETR pode estar correlacionada à taxa fotossintética bruta $\left(\mathrm{A}_{\mathrm{G}}\right)$, apresentando o mesmo padrão da curva de assimilação de $\mathrm{CO}_{2}$. Neste estudo, a curva de assimilação de $\mathrm{CO}_{2}$ não foi realizada nas quatro espécies, mas a tendência das curvas de ETR sugere que as taxas fotossintéticas tenham reduzido concomitantemente com o transporte de elétrons. 
Tabela 2 - Valores médios do rendimento quântico máximo $\left(F_{v} / F_{m}\right)$ em quatro espécies de gramíneas forrageiras tropicais com até dez dias de suspensão da irrigação

Tabela 2 - Mean values of maximum quantum yield $\left(F_{\checkmark} / F_{m}\right)$ determined in four tropical forage grasses, during ten days of interuption of irrigation

\begin{tabular}{|c|c|c|c|c|c|}
\hline & \multicolumn{5}{|c|}{$\begin{array}{l}\text { Dias após suspensão da irrigação } \\
\text { Days after interruption of irrigation }\end{array}$} \\
\hline & 2 & 4 & 6 & 8 & 10 \\
\hline Capim-hemárthria (Hemarthriagrass) & $0,751 \mathrm{aA}$ & $0,714 \mathrm{aA}$ & $0,744 \mathrm{aA}$ & $0,731^{\mathrm{a}} \mathrm{A}$ & $0,697^{\mathrm{a}} \mathrm{A}$ \\
\hline Capim-angola (Angolagrass) & $0,676 \mathrm{aB}$ & $0,683 \mathrm{aA}$ & $0,672 \mathrm{aB}$ & $0,632 \mathrm{abB}$ & $0,595 \mathrm{bB}$ \\
\hline Capim-do-nilo (Nilograss) & $0,652 \mathrm{aB}$ & $0,606 \mathrm{aB}$ & $0,647 \mathrm{aB}$ & $0,111 \mathrm{bD}$ & $0,100 \mathrm{bC}$ \\
\hline
\end{tabular}

Médias seguidas das mesmas letras, maiúsculas na coluna e minúsculas na linha, não diferem $(P>0,05)$ pelo testeTukey.

Means followed by the same letter, capital in columns and small in a row, do not differ $(P>0.05)$, by Tukey test.

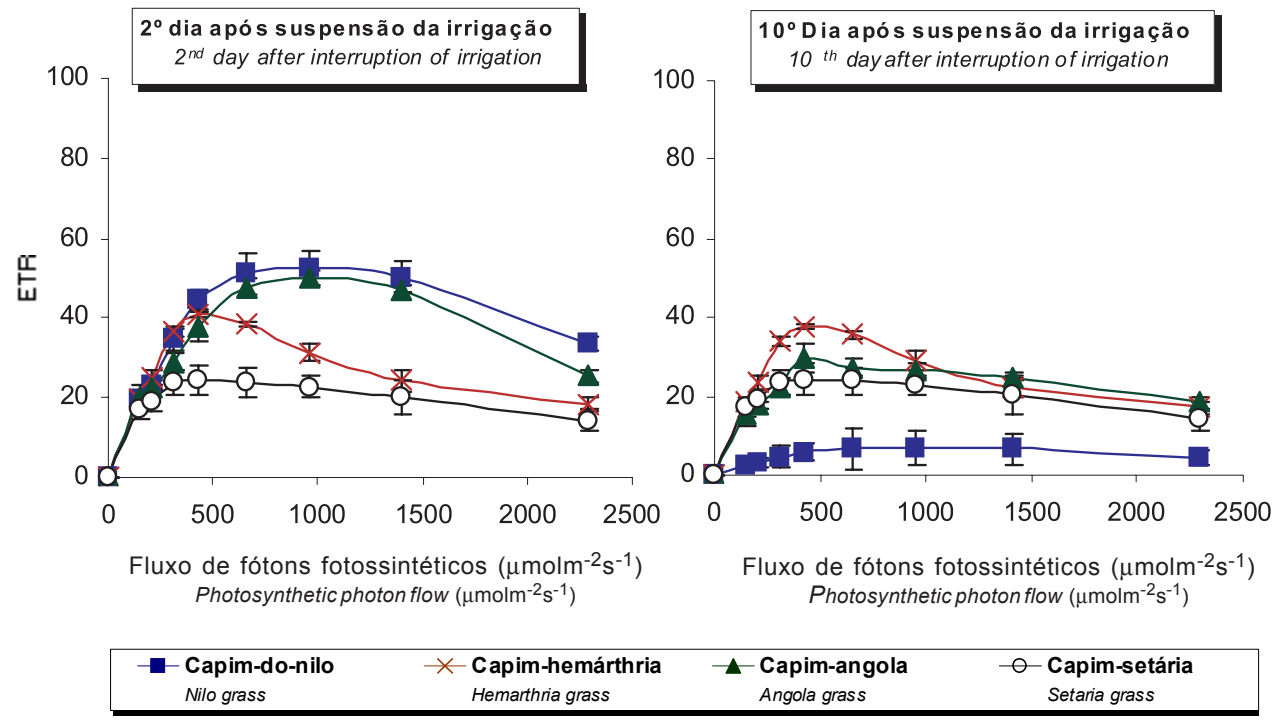

Figura 2 - Efeito do fluxo de fótons fotossintéticos sobre as curvas da taxa relativa de transporte de elétrons (ETR) em quatro espécies de gramíneas forrageiras tropicais mantidas sob déficit hídrico. As medições foram realizadas após 30 minutos da adaptação das folhas ao escuro. As barras em cada ponto das curvas representam o erro-padrão da média de três repetições.

Figure 2 - Curves of relative electron transport rate (ETR) in response to photosynthetic photon flow in four species of tropical forage grasses, maintained under conditions of total hydric saturation of soil porosity. The measurements were accomplished 30 minutes after dark adaptation. The bars to each point in the curves represent the standard error of mean of three replicates.

As respostas das variáveis da fluorescência da clorofila $a$ revelam o nível de excitação de energia dos pigmentos e são dependentes do balanço entre o fluxo de fótons fotossintéticos (FFF) e a soma da taxa de transporte de elétrons (ETR) fotossintético e da dissipação térmica, o que pode ser estimado pelo quenching fotoquímico (qP) e nãofotoquímico (qN). O aumento do FFF promove declínio no $\mathrm{qP}$ e aumento no qN. O decréscimo do qP reflete o estado reduzido do primeiro aceptor de elétrons estável do PSII, a $\mathrm{Q}_{\mathrm{A}}$, fornecendo uma estimativa da capacidade do PSII em utilizar a energia luminosa para redução do $\mathrm{NADP}^{+}$, indispensável à assimilação fotossintética do carbono. Por sua vez, o qN indica a eficiência da dissipação de calor, em razão do aumento no gradiente de prótons entre o lúmen e o estroma do cloroplasto (Genty et al., 1989; Maxwell \& Johnson, 2000).

Verificaram-se variações nos valores dos quenchings entre as gramíneas, em decorrência do fluxo de fótons fotossintéticos, a partir do segundo dia de suspensão da irrigação nas plantas (Figura 3). Na análise do qP, foi evidente o padrão de queda dessa variável quando as folhas intactas foram expostas ao pulso saturante, indicando que a plastoquinona $\mathrm{Q}_{\mathrm{A}}$ (primeiro aceptor de elétrons estável do PSII) estava completamente reduzida. Pode-se inferir, portanto, que o centro de reação do PSII se encontrava em estado "fechado", podendo-se determinar a "pressão de excitação" no PSII (Maxwell et al., 1994). Os valores observados dos quenchings apontaram para menores qP no capim-do-nilo e capim-setária quando avaliados no $10^{\mathrm{O}}$ dia após a suspensão da irrigação, em comparação ao início das avaliações (Figura 3), ou seja, sob maior déficit hídrico estas espécies parecem apresentar indícios de danos às proteínas do complexo fotossintético. Mesmo sob déficit hídrico intenso, os capins angola e hemárthria mantiveram valores de $\mathrm{qP}$ elevados e, portanto, parecem ser menos susceptíveis ao estresse hídrico experimental. 

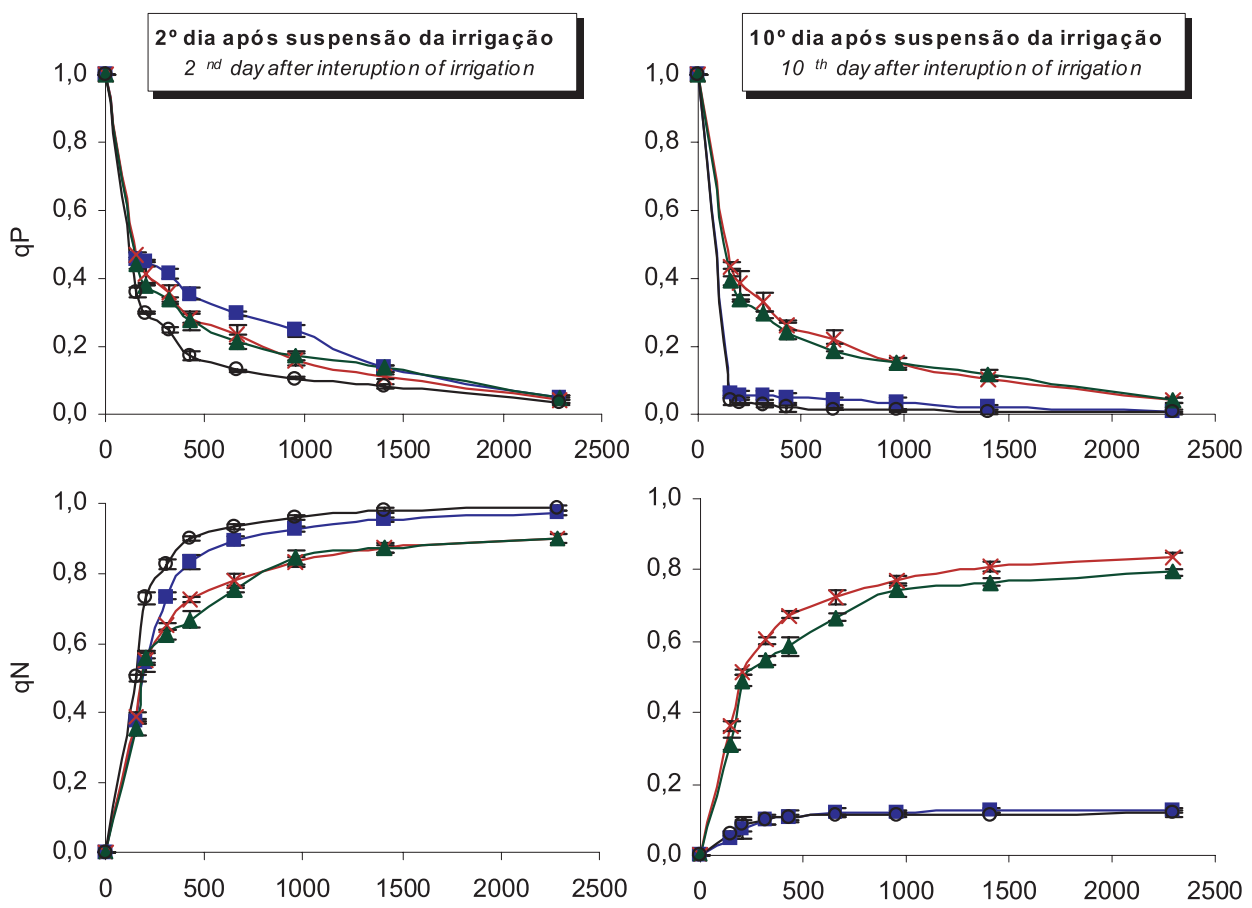

Fluxo de fótons fotossintéticos $\left(\mu \mathrm{molm}^{-2} \mathrm{~s}^{-1}\right)$ Photosynthetic photon flow $\left(\mu \mathrm{molm}^{-2} \mathrm{~s}^{-1}\right)$

Fluxo de fótons fotossintéticos $\left(\mu \mathrm{molm}^{-2} \mathrm{~s}^{-1}\right)$ Photosynthetic photon flow $\left(\mu \mathrm{molm}^{-2} \mathrm{~s}^{-1}\right)$

\begin{tabular}{|cccc|}
\hline $\begin{array}{c}- \text { Capim-do-nilo } \\
\text { Nilo grass }\end{array}$ & $\times$ Capim-hemárthria & - Capim-angola & - Angola grass \\
Hemarthria grass & Capim-setária & Setaria grass \\
\hline
\end{tabular}

Figura 3 - Efeito do fluxo de fótons fotossintéticos sobre os quenchings fotoquímico (qP) e não-fotoquímico (qN) de fótons fotossintéticos em quatro espécies de gramíneas forrageiras tropicais mantidas sob déficit hídrico. As medições foram realizadas após 30 minutos da adaptação das folhas ao escuro. As barras em cada ponto das curvas representam o erro-padrão da média de três repetições.

Figure 3 - Photochemical quenching $(q P)$ and non-photochemical quenching $(q N)$ in response to photosynthetic photon flux in four species of tropical forage grasses, maintained under conditions of total hydric saturation of porosity of soil. The measurements were done 30 minutes after dark adaptation. The bars to each point in the curves representing the standard-error of mean of three replicates.

Observaram-se menores valores de qN no capim-donilo e no capim-setária e maiores nos capins hemárthria e angola. O qN verificado nas quatro espécies no segundo dia sem irrigação foi próximo ao valor máximo $(1,0)$. No décimo dia após a suspensão da irrigação, o valor qN reduziu significativamente no capim-do-nilo e no capimsetária, indicando que provavelmente o aparelho fotossintético dessas espécies poderia estar severamente danificado pela ocorrência de fotoinibição. Entretanto, na mesma condição de avaliação, os capins hemárthria e angola mantiveram valores de qN elevados, de 0,83 e 0,79, respectivamente, o que indica provável regulação fotoprotetora, possibilitada pela capacidade de dissipação da energia absorvida em excesso, seja pelo aumento do gradiente de prótons entre o lúmen e o estroma do cloroplasto, seja pela ação da zeaxantina (Maxwell \& Johnson, 2000). Estes resultados poderiam refletir o desempenho dessas duas espécies, indicando maior capacidade de tolerar as condições fotoinibitórias ocasionadas pelo estresse hídrico.

\section{Conclusões}

Com o aumento do estresse hídrico, ocorreu diminuição nos valores de $F_{0}$, da $F_{m}$ e da taxa $F_{v} / F_{m}$ nos capins estudados. Entretanto, estas reduções foram mais acentuadas para o capim-do-nilo, que apresentou, concomitantemente, características de senescência das folhas mais evidentes a partir do quarto dia sem irrigação.

A capacidade de tolerar o déficit hídrico pode estar associada à maior eficiência do sistema fotoprotetor do PSII, que pode ser atribuída ao capim-hemárthria, em razão de seu melhor desempenho fotoquímico em relação às demais gramíneas avaliadas.

\section{Literatura Citada}

ALCÂNTARA, P.B.; BUFARAH, G. Plantas forrageiras: gramíneas e leguminosas. 4.ed. São Paulo: Nobel, 1992. $162 \mathrm{p}$.

ARMSTRONG, W.; JUSTIN, S.H.F.W.; BECKETT, P.M. et al. Root adaptation to soil waterlogging. Aquatic Botany, v.39, p.57-73, 1991. 
BAKER, N.R. A possible role for photosystem II in environmental perturbations of photosynthesis. Physiologia Plantarum, v. 81, n.4, p.563-70, 1991.

BARUCH, Z. Responses to drought and flooding in tropical forage grasses. 2. Leaf water potential, photosynthesis rate and alcohol dehydrogenase activity. Plant Soil, v.164, p.97-105, 1994.

BJÖRKMAN, O.; DEMMING, B. Photon yield of $\mathrm{O}_{2}$ evolution and chlorophyll fluorescence characteristics at $77 \mathrm{k}$ among vascular plants of diverse origins. Planta, v.170, p.61-66, 1987.

BOLHÀR-NORDENKAMPF, H.R.; LONG, S.P., BAKER, N.R., et al. Chlorophyll fluorescence as probe of the photosynthetic competence of leaves in the field: a review of current instrument. Functional Ecology, v.3, p.497-514, 1989.

BULKHOV, N.; WIESE, C.; NEIMANIS, S. et al. Heat sensitivity of chloroplasts and leaves: Leakage of protons from thylakoids and reversible activation of cyclic eletron transport. Photosynthesis Research, v.59, p.81-93, 1999.

COSTA, E.S; BRESSAN-SMITH, R.; OLIVEIRA, J.G.; CAMPOSTRINI, E. Chlorophyll $a$ fluorescence analysis in response to excitation irradiance in bean plants (Phaseolus vulgaris L. and Vigna unguiculata L. Walp) submitted to high temperature stress. Photosynthetica, v.41, p.77-82, 2003.

DE LAS RIVAS, J.; BARBER, J. Structure and thermal stability of photo system II reaction centers studied by infrared spectroscopy. Biochemistry, v.36, p.8897-8903, 1997.

DREW, M.C.; JACKSON, M.B.; GIFFARD, S. Ethylene promoted adventitious rooting and development of cortical air spaces (aerenchyma) in roots may be adaptative responses to flooding in Zea mays L. Planta, v.147, p.83-88, 1979.

DURÃES, F.O.M. 2003. Fotossíntese e fluorescência da clorofila: Parâmetros indicadores de tolerância a estresses. Disponível em: www.cnpms.embrapa.br/nucleos/nea/pesquisa/ gest002.html. Acesso em: em 04/08/03.

EUCLYDES, R.F. Sistema para análises estatísticas e genéticas (SAEG) - manual prático. Viçosa, MG: Universidade Federal de Viçosa, 1983. 74p.

GATES, D.M. Transpiration and leaf temperature. Annual Review Plant Physiology, v.19, p.211-238, 1968.

GENTY, B.; BRIANTAIS, J.M.; BAKER, N. The relationship between the quantum yield of photosynthetic electron transport and quenching chlorophyll fluorescence. Biochimica et Biophysica Acta, v.990, p.87-92, 1989.
HORTON, P.; RUBAN, A.V.; WALTERS, R.G. Regulation of light harvesting in green plants. Annual Review Plant Physiology, Plant Molecular Biology, v.47, p.655-684, 1996.

KAISER, W.M. Effect of water deficit on photosynthetic capacity. Physiologia Plantarum, v.71, p.142-49, 1987.

KIEHL, E.J. Manual de edafologia - Relações solo-planta. São Paulo: Agronômica Ceres, 1979. 264p.

KRAUSE, G.H.; WEIS, E. Chlorophyll fluorescence and photosynthesis: the basics. Annual Review Plant Physiology. Plant Molecular Biology, v.42, p.313-349, 1991.

LARCHER, W. Physiological plant ecology. 3.ed. New York: Springer-Verlag Berlin Heidelberg, 1995. 340p.

MAXWELL, D.P.; FALK, S.; TRICK, C.G. et al. Growth at low temperature mimics high-light acclimation in Chlorella vulgaris. Plant Physiology, v.105, n.2, p.947-957, 1994.

MAXWELL, K.; JOHNSON, G.N. Chlorophyll fluorescence - a practical guide. Journal of Experimental Botany, v. 51 , n.345, p.659-668, 2000

MOHAMED, G.H.; BINDER, W.D.; GILLES, L. Chlorophyll fluorescence: a review of its practical foresty applications and instrumentation. Scandinavian Journal of Forage Research, v. 10, p.383-410, 1995.

RHING, J.M.L.C.; GOODENOUGH, D.C.W. Acroceras macrum Stapf (Nilegrass) - a review. Proceedings of the Grassland Society of Southern Africa, v.14, p.27-36, 1979.

RODRIGUES, L.R.A.; RODRIGUES, T.J.D. Ecofisiologia de plantas forrageiras. In: CASTRO, P.R.C.; FERREIRA, S.O.; YAMADA, T. (Eds.) Ecofisiologia da produção agrícola. Piracicaba: Fundação de Estudos Agrários Luiz de Queiroz, 1994. p.203230.

SILVA, S.; SOARES, A.M.; OLIVEIRA, L.E.M. et al. Respostas fisiológicas de gramíneas promissoras para revegetação ciliar de reservatórios hidrelétricos, submetidos à deficiência hídrica. Ciência e Agrotecnologia, v.25, n.1, p.124-133, 2001.

WERNER, J.C.; PAULINO, V.T.; CANTARELLA, H. et al. Recomendações de adubação e calagem para o Estado de São Paulo. 2.ed. Campinas: Instituto Agronômico de Campinas, 1986. p.263-273. 